\title{
BRAZILIAN SANDY BEACH MACROFAUNA PRODUCTION: A REVIEW
}

\author{
Marcelo Petracco $^{l, *}$, Ricardo Silva Cardoso ${ }^{2}$, Thais Navajas Corbisier ${ }^{l}$ and Alexander Turra ${ }^{l}$ \\ ${ }^{1}$ Instituto Oceanográfico da Universidade de São Paulo \\ (Praça do Oceanográfico, 191, 05508-120 São Paulo, SP, Brasil) \\ ${ }^{2}$ Universidade Federal do Estado do Rio de Janeiro (UNIRIO) \\ Instituto de Biociências - Departamento de Ecologia e Recursos Marinhos \\ (Av. Pasteur, 458, 22290-240 Rio de Janeiro, RJ, Brasil) \\ *Corresponding author: mpetracco@uol.com.br
}

\begin{abstract}
A B S TR A C T
The state of the art of the studies on the production of Brazilian sandy beach macrofauna was analyzed on the basis of the data available in the literature. For this purpose, the representativeness of the production dataset was examined by latitudinal distribution, degree of exposure and morphodynamic state of beaches, taxonomic groups, and methods employed. A descriptive analysis was, further, made to investigate the trends in production of the more representative taxonomic groups and species of sandy beach macrofauna. A total of 69 macrofauna annual production estimates were obtained for 38 populations from 25 studies carried out between $22^{\circ} 56^{\prime} \mathrm{S}$ and $32^{\circ} 20^{\prime} \mathrm{S}$. Production estimates were restricted to populations on beaches located on the southern and southeastern Brazilian coast. Most of the populations in the dataset inhabit exposed dissipative sandy beaches and are mainly represented by mollusks and crustaceans, with a smaller number of polychaetes. The trends in production among taxonomic groups follow a similar pattern to that observed on beaches throughout the world, with high values for bivalves and decapods. The high turnover rate $(\mathrm{P} / \mathrm{B}$ ratio) of the latter was due to the presence of several populations of the mole crab Emerita brasiliensis, which can attain high values of productivity, in the dataset. Most of the studies focus on the comparison of production and, especially, of $\mathrm{P} / \mathrm{B}$ ratio according to life history traits in populations of the same species/taxonomic group. Despite the importance of life history-production studies, other approaches, such as the effect of man-induce disturbances on the macrofauna, should be undertaken in these threatened environments.
\end{abstract}

\section{R ESUMO}

O estado da arte dos estudos de produção da macrofauna de praias arenosas brasileiras foi analisado a partir de informações disponíveis na literatura. Para essa finalidade, a representatividade dos dados de produção foi examinada de acordo com a distribuição latitudinal, grau de exposição e estado morfodinâmico das praias, grupos taxonômicos e métodos empregados. Além disso, foi realizada uma análise descritiva para investigar tendências da produção dos grupos taxonômicos e das espécies mais representativas da macrofauna de praias arenosas. Um total de 69 estimativas de produção referentes a 38 populações da macrofauna foi obtido a partir de 35 estudos realizados entre $22^{\circ} 56^{\prime} \mathrm{S}$ e $32^{\circ} 20^{\prime} \mathrm{S}$. Estimativas de produção foram restritas a populações de praias situadas no sul e sudeste da costa brasileira. A maior parte das populações estudadas habita praias arenosas expostas dissipativas e é representada principalmente por moluscos e crustáceos, com um número menor de poliquetas. As tendências da produção entre os grupos taxonômicos seguiram um padrão similar ao observado em praias ao longo do mundo, com altos valores para bivalves e decápodes. A alta taxa de renovação (razão P/B) dos decápodes foi devido à presença de diversas estimativas para populações do caranguejo Emerita brasiliensis, as quais alcançam altos valores de produtividade. A maior parte dos estudos aborda a comparação da produção e, especialmente, da razão P/B, de acordo com características da história de vida de populações da mesma espécie e/ou com grupo taxonômico. Apesar da importância de estudos com esse enfoque, outras abordagens, tais como o efeito de distúrbios induzidos pelo homem sobre a macrofauna, deveriam ser empregadas nesses ambientes ameaçados.

Descriptors: Sandy beaches, Macrofauna, Secondary production, P/B ratio, Brazil. Descritores: Praias arenosas, Macrofauna, Produção secundária, Razão P/B, Brasil. 


\section{INTRODUCTION}

Secondary production is a flow which represents the formation of biomass through the growth of an individual, or collectively in terms of a population, over time (BENKE, 2010). This variable is a composite measure of density, biomass, growth rate, reproduction, survivorship, and development time (BENKE, 1993) and is, therefore, a key parameter in population ecology (SARDÁ et al., 2000). Production analysis also provides a common link between populations and ecosystems as it represents a measure of the population's function at community/ecosystem level (BENKE, 1993). Despite the importance of this functional variable and its several applications to ecological issues, it is often addressed but briefly in most ecology texts and is usually limited to the context of energy flow and trophic-level efficiencies (BENKE, 2010). Moreover, the assessment of secondary production requires expensive and time consuming sampling programs, even at the level of a single population (DOWNING, 1984; BREY, 1990; CUSSON; BOURGET, 2005). Thus, despite the increase in the number of secondary production studies in recent years, this variable is still under-used (BENKE; HURYN, 2010).

Sandy beaches dominate the world's temperate and tropical shorelines and are generally characterized by low production, both primary and secondary (DEXTER, 1992; McLACHLAN; BROWN, 2006). However, very high values of secondary production have been estimated for sandy beach macrofauna populations (e.g. GIANUCA, 1985; LAUDIEN et al., 2003). Moreover, Wilson (1999) has indicated the importance of sandy beaches as exporters of productivity to adjacent ecosystems, and also to total coastal productivity due to the great extension of these ecosystems. Therefore, it is important to obtain estimates of sandy beach macrofauna production, especially in Brazil, which has an extensive coastline. Furthermore, species of the sandy beach macrofauna are harvested extensively around the world and play a very important role as food sources for several marine and terrestrial species, including fish and crustaceans of commercial interest (PETERSON et al., 2000; McLACHLAN; BROWN, 2006).

According to what is generally observed around the world, Brazilian sandy beaches are frequently subject to various forms of man-induced stress such as oil spills, contaminated freshwater discharges, structures or activities affecting natural sand transport, and balance, among others, which can affect the life history traits of the macrofauna populations of these ecosystems (AMARAL et al., 2003; SCHLACHER et al., 2007; DUGAN et al., 2008; DEFEO et al., 2009). Therefore, estimates of secondary production are a valuable tool for the rational management of aquatic resources and the evaluation of the effects of man-induced stress (DOWNING, 1984; BENKE; HURYN, 2010).

In Brazil, several studies, since Gianuca's (1985) pioneering work, have obtained production estimates for macrofauna. The resulting update in knowledge of Brazilian beach macrofauna studies makes it possible to identify major gaps in knowledge and establish guidelines as well as encouraging future studies in a still incipient, but very important subject. The aim of this study is, therefore, to review the studies on production of sandy beach macrofauna in Brazil, and, specifically: 1) evaluate the representativeness of the production data according to the geographical/latitudinal distribution, degree of exposure and morphodynamic state of beaches, taxonomic groups, and the methods employed, 2) analyze the trends in production and productivity $(\mathrm{P} / \mathrm{B}$ ratio) of the more representative taxonomic groups and species in the dataset, and 3) analyze the approaches of the production studies on Brazilian sandy beach macrofauna.

\section{Dataset Collection}

A broad literature review on production of Brazilian sandy beach macrofauna was carried out, considering the values of annual production, mean annual biomass, and the annual P/B ratio. Macrofauna estimates in other intertidal habitats were not included in the dataset. Life span and mean body mass were culled from each study because they are the biotic variables which are most frequently related to the $\mathrm{P} / \mathrm{B}$ ratio (ROBERTSON, 1979; BREY, 1990; HURYN; BENKE, 2007). Life span was generally given in the studies, while the mean body mass was generally calculated by dividing the mean annual biomass by the mean annual density (CUSSON; BOURGET, 2005). The instantaneous mortality rate $(\mathrm{Z})$ was obtained from each study in the light of Allen's relationship $(\mathrm{Z}=\mathrm{P} / \mathrm{B})$ (ALLEN, 1971). The methods used to estimate the annual production and the $\mathrm{P} / \mathrm{B}$ ratio were recorded and classified as either cohort or size-based methods (see BENKE, 1984, 1993; BENKE; HURYN, 2006 for theoretical background).

The latitude of the study area and its environmental variables, beach exposure (sheltered or exposed beach sensu McLachlan, 1980), and the morphodynamic state of the beach (dissipative, intermediate, and reflective sensu Short, 1996) were collected. Production and biomass data expressed in running meters $\left(\mathrm{m}^{-1}\right)$ were transformed into square meters dividing these values by population distribution width in the intertidal zone. When necessary, production, biomass, and mean body mass were converted to ash-free dry mass (AFDM) using the conversion factors of Ricciardi and Bourget (1998), 
Brey et al. (1988), and Brey (2001). A descriptive analysis was performed to compare the number of populations studied by geographical/latitudinal distribution, degree of exposure and morphodynamic state of beaches, taxonomic group, and method employed. The statistical analysis was not employed to compare production and productivity between the taxonomic groups due to the paucity of data available in the literature reviewed. Therefore, a descriptive analysis was performed to analyze the trends in production and productivity of the more representative taxonomic groups and species in the dataset. If more than one classical method (cohort and size-based) was employed to estimate the annual production for a population, mean estimates of production and $\mathrm{P} / \mathrm{B}$ ratio were calculated for each population. However, when the population production was estimated for more than one year (for example, by a two-year sampling), all annual production, mean annual biomass, and $\mathrm{P} / \mathrm{B}$ ratios values were considered. The median and mean production, biomass, $\mathrm{P} / \mathrm{B}$ ratio, life span, and mean body mass for each taxonomic group were calculated.

Representativeness, General Characteristics, and Accuracy of the Dataset

All the production data collected from the literature as well as data on the biological and environmental variables on Brazilian sandy beaches are shown in Table 1. A total of 69 annual production estimates were obtained for 38 macrofauna populations from 25 studies carried out between $22^{\circ} 56^{\prime} \mathrm{S}$ and $32^{\circ} 20^{\prime} \mathrm{S}$. The cumulative frequency of the number of populations of Brazilian sandy beach macrofauna studied shows a continuous increase in the number of studies since early 2000 (Fig. 1).

Most of the populations studied inhabit beaches between latitudes 21 and $25^{\circ} \mathrm{S}$ (Fig. 2a). Regarding the degree of exposure, more populations from exposed beaches (23) than from sheltered ones (16) were studied. According to the morphodynamic state of exposed beaches, a greater number of populations were studied on dissipative (12) than on intermediate (6) and reflective beaches (6) Populations were distributed in the following taxonomic groups: Mollusca (18) (Bivalvia (9), Gastropoda (9)), Crustacea (15) (Decapoda (9), Isopoda (5), Amphipoda (1)), and Polychaeta (6) (Fig. $2 \mathrm{~b}$, c and Table 1). Most populations are represented by isopods of the genus Excirolana (5), decapods of the species Emerita brasiliensis(4), polychaetes of the genus Scolelepis (4), and bivalves of the species Anomalocardia brasiliana (4) (Table 1).

In view of the general scarcity of production estimates for sandy beach macrofauna, there was a reasonable number of studies on the production of
Brazilian sandy beach macrofauna. For instance, most of the studies on polychaete, decapod, and gastropod production on beaches around the world were carried out on the Brazilian coast. Moreover, the number of recent studies published (e.g. MATTOS; CARDOSO, 2012; OTEGUI et al., 2012; PETRACCO et al., 2012a), shows that the studies on sandy beach macrofauna production in Brazil (Fig. 1) tend to increase.

In contrast with the number of populations studied in southern and southeastern Brazil (21 to $32^{\circ} \mathrm{S}$ ), none of the published studies consider the production of species on beaches located on the northeastern and northern coast of Brazil (Fig. 2a), which was unexpected. The absence of studies on such an extensive stretch of the Brazilian coast is quite troubling given the importance of production estimates for the macrobenthos. Most populations inhabit exposed beaches, especially the dissipative ones located on the coast of the states of Rio Grande do Sul, Paraná and the southern coast of São Paulo (GIANUCA, 1985; SOUZA et al., 1998; SOUZA; BORZONE, 2000, 2007; PETRACCO et al., 2012a). The studies on the populations of reflective exposed beaches relate to the coast of Rio de Janeiro (CARDOSO; VELOSO, 1996; VELOSO et al., 2003; PETRACCO et al., 2003; CAETANO et al., 2006; VELOSO; SALLORENZO, 2010). The majority of the few studies on the populations of sheltered beaches throughout the world have been carried out in Brazil. These studies are mainly on the mollusk populations of beaches located in southeastern Brazil, on the coast of São Paulo (e.g. DENADAI et al., 2004) and Rio de Janeiro (e.g. CABRINI; CARDOSO, 2010, 2012; MATTOS; CARDOSO, 2012) (Table 1 and Figure $2 b)$.

Bivalvia is the most studied group worldwide with regard to its production probably due to the fact that the species of this group are frequently harvested and used as food or income sources (McLACHLAN et al., 1996; HERRMANN et al., 2009). However, the number of bivalve populations studied on Brazilian beaches, is equal to that of those on gastropods and decapods (Fig. 2c and Table 1). Of the eight populations of sandy beach polychaetes studied worldwide, six relate to the Brazilian coast. This shows that the scarcity of studies on this group is not only true of Brazilian sandy beaches. As for other marine environments (see CUSSON; BOURGET, 2005), only a small number of Brazilian sandy beach isopod populations have been studied. However, unlike what has been observed regarding marine habitats in general, and specifically for sandy beaches, the knowledge of the amphipod production on Brazilian sandy beaches is restricted to a single population (CARDOSO; VELOSO, 1996). 
Table 1. Characterization of biological and physical attributes of the dataset of Brazilian sandy beach macrofauna in different latitudes (Lat). Annual production (P: $\mathrm{g}_{\mathrm{AFDM} \mathrm{m}}^{-2} \mathrm{yr}^{-1}$ ), mean annual biomass (B: $\mathrm{g}_{\mathrm{AFDM} \mathrm{m}}{ }^{-2}$ ), annual turnover rate (P/B: year $^{-1}$ ), life span (LS: years), mean body mass $\left(\mathrm{M}_{\text {mean }}\right.$ g AFDM), and instantaneous mortality rate $\left(\mathrm{Z}\right.$ : $\left.\mathrm{yr}^{-1}\right)$. Environmental characteristics of sandy beaches: degree of exposure (DE): exposed (E) and sheltered (S), morphodynamic state of exposed beaches (MS): dissipative (D), intermediate (I) and reflective (R); mean annual seawater temperature $\left(\mathrm{T}\right.$ : $\mathrm{C}^{\circ}$ ). Methods of production employed (Met): cohort methods (increment summation (IS) and removal summation (RS) methods), size-based methods (mass-specific growth rate (MSGR) and size-frequency (SF) methods).

\begin{tabular}{|c|c|c|c|c|c|c|c|c|c|c|c|}
\hline $\begin{array}{l}\text { Group/Species } \\
\text { Mollusca }\end{array}$ & $\mathbf{P}$ & B & $\mathbf{P} / \mathbf{B}$ & $\mathbf{L S}$ & $\mathbf{M}_{\text {mean }}$ & $\mathbf{Z}$ & Met & DE & MS & Lat & Source \\
\hline \multicolumn{12}{|l|}{ Bivalvia } \\
\hline $\begin{array}{l}\text { Anomalocardia } \\
\text { brasiliana }\end{array}$ & 11.60 & 9.14 & 1.27 & 2.64 & & 3.17 & MSGR & $\mathrm{S}$ & & $22^{\circ} 56^{\prime} \mathrm{S}$ & $\begin{array}{l}\text { (MATTOS; CARDOSO, } \\
\text { 2012) }\end{array}$ \\
\hline Diplotonda patagonica & 0.79 & 0.43 & 1.82 & 1.63 & & 3.53 & MSGR & $\mathrm{s}$ & & $22^{\circ} 56^{\prime} \mathrm{S}$ & $\begin{array}{l}\text { (MATTOS; CARDOSO, } \\
\text { 2012) }\end{array}$ \\
\hline & & & & & & & & & & & (CARDOSO; VELOSO, \\
\hline Donax hanleyanus & 0.76 & 0.48 & 1.59 & 1.44 & 0.01632 & 1.70 & MSGR & E & I & $23^{\circ} 03^{\prime} \mathrm{S}$ & $\begin{array}{l}\text { 2003) } \\
\text { (CARDOSO; VELOSO, }\end{array}$ \\
\hline $\begin{array}{l}\text { Donax hanleyanus } \\
\text { Anomarlocardia }\end{array}$ & 3.67 & 2.53 & 1.45 & 1.47 & 0.01549 & 1.55 & MSGR & E & I & $23^{\circ} 03^{\prime} \mathrm{S}$ & 2003) \\
\hline brasiliana & 5.45 & 1.96 & 2.78 & 2.00 & 0.03704 & 3.61 & MSGR & $\mathrm{S}$ & & $23^{\circ} 37^{\prime} \mathrm{S}$ & ${ }^{(1)}$ (CORTE, 2011) \\
\hline Anomarlocardia & 1.47 & 079 & 185 & 200 & 005684 & 141 & MSGR & $S$ & & $23^{\circ} 45^{\prime} \mathrm{S}$ & (2)(SALVADOR 2001) \\
\hline Corbula cubaniana & $\begin{array}{l}1.47 \\
0.06\end{array}$ & 0.03 & $\begin{array}{l}1.85 \\
1.87\end{array}$ & $\begin{array}{l}2.00 \\
4.00\end{array}$ & $\begin{array}{l}0.00084 \\
0.00639\end{array}$ & $\begin{array}{l}1.41 \\
1.88\end{array}$ & MSGR & $\begin{array}{l}3 \\
S\end{array}$ & & $\begin{array}{l}23 \text { 4S S } \\
23^{\circ} 45^{\prime} \mathrm{S}\end{array}$ & (2) (SALVADOR, 2001) \\
\hline \multicolumn{12}{|l|}{ Anomarlocardia } \\
\hline brasiliana & 0.59 & 0.66 & 0.89 & 3.00 & 0.01241 & 0.97 & MSGR & $\mathrm{s}$ & & $23^{\circ} 45^{\prime} \mathrm{S}$ & (1)(CORTE, 2011) \\
\hline Mesodesma mactroides & 185.30 & 107.15 & 1.70 & 5.00 & & & IS & E & $\mathrm{D}$ & $32^{\circ} 20^{\prime} \mathrm{S}$ & (GIANUCA, 1985) \\
\hline Donax hanleyanus & 13.56 & 3.45 & 3.90 & & 0.00123 & & IS & $\mathrm{E}$ & $\mathrm{D}$ & $32^{\circ} 20^{\prime} \mathrm{S}$ & (GIANUCA, 1985) \\
\hline \multicolumn{12}{|l|}{ Gastropoda } \\
\hline Cerithium atratum & 4.56 & 3.90 & 1.17 & 4.26 & 0.01950 & 4.10 & MSGR & S & & $22^{\circ} 57^{\prime} \mathrm{S}$ & $\begin{array}{l}\text { (CABRINI; CARDOSO, } \\
2010)\end{array}$ \\
\hline Nassarius vibex & 0.67 & 0.58 & 1.16 & 2.58 & 0.02762 & 4.20 & MSGR & $\mathrm{S}$ & & $22^{\circ} 57^{\prime} \mathrm{S}$ & $\begin{array}{l}\text { (CABRINI; CARDOSO, } \\
\text { 2012) }\end{array}$ \\
\hline Olivancillaria vesica & 0.17 & 0.17 & 1.03 & 4.28 & $\begin{array}{l}.02702 \\
1.07139\end{array}$ & $\begin{array}{l}4.20 \\
3.12\end{array}$ & MSGR & $\mathrm{E}$ & I & $23^{\circ} 03^{\prime} \mathrm{S}$ & (CAETANO et al., 2003) \\
\hline Olivancillaria vesica & 0.14 & 0.13 & 1.11 & 4.28 & 0.98016 & 3.12 & SF & $\mathrm{E}$ & I & $23^{\circ} 03^{\prime} \mathrm{S}$ & (CAETANO et al., 2003) \\
\hline Olivancillaria vesica & 0.21 & 0.20 & 1.06 & 4.99 & 1.07139 & 2.56 & MSGR & $\mathrm{E}$ & I & $23^{\circ} 03^{\prime} \mathrm{S}$ & (CAETANO et al., 2003) \\
\hline Olivancillaria vesica & 0.15 & 0.14 & 1.05 & 4.99 & 0.98016 & 2.56 & SF & $\mathrm{E}$ & I & $23^{\circ} 03^{\prime} \mathrm{S}$ & (CAETANO et al., 2003) \\
\hline Nassarius vibex & 0.35 & 0.90 & 0.38 & 4.00 & 0.02930 & 0.92 & MSGR & S & & $23^{\circ} 37^{\prime} \mathrm{S}$ & (3) (YOKOYAMA, 2010) \\
\hline Nassarius vibex & 3.60 & 7.98 & 0.45 & 5.00 & 0.02474 & 1.01 & MSGR & S & & $23^{\circ} 37^{\prime} \mathrm{S}$ & (3) (YOKOYAMA, 2010) \\
\hline Cerithium atratum & 1.15 & 0.29 & 3.90 & 1.60 & 0.00370 & 2.67 & IS & S & & $23^{\circ} 47^{\prime} \mathrm{S}$ & (DENADAI et al., 2004) \\
\hline Olivella minuta & 0.07 & 0.04 & 1.58 & 2.97 & 0.00216 & 4.37 & MSGR & S & & $23^{\circ} 47^{\prime} \mathrm{S}$ & $\begin{array}{l}\text { (PETRACCO et al., } \\
\text { unpublished data) } \\
\text { (PETRACCO et al., }\end{array}$ \\
\hline Olivella minuta & 0.07 & 0.04 & 1.58 & 2.97 & 0.00216 & 4.37 & $\mathrm{SF}$ & S & & $23^{\circ} 47^{\prime} \mathrm{S}$ & unpublished data) \\
\hline Olivella minuta & 0.03 & 0.03 & 1.19 & 2.77 & 0.00622 & 6.24 & MSGR & S & & $23^{\circ} 49^{\prime} \mathrm{S}$ & unpublished data) \\
\hline Olivella minuta & 0.03 & 0.03 & 1.20 & 2.77 & 0.00622 & 6.24 & SF & $\mathrm{s}$ & & $23^{\circ} 49^{\prime} \mathrm{S}$ & $\begin{array}{l}\text { (PETRACCO et al., } \\
\text { unpublished data) }\end{array}$ \\
\hline Hastula cinerea & 0.19 & 0.15 & 1.26 & 3.13 & 0.00731 & 3.78 & MSGR & $\mathrm{E}$ & $\mathrm{D}$ & $24^{\circ} 27^{\prime} \mathrm{S}$ & (4) (PETRACCO, 2008) \\
\hline Hastula cinerea & 0.19 & 0.15 & 1.30 & 3.13 & 0.00731 & 3.78 & SF & $\mathrm{E}$ & $\mathrm{D}$ & $24^{\circ} 27^{\prime} \mathrm{S}$ & (4) (PETRACCO, 2008) \\
\hline Hastula cinerea & 0.11 & 0.16 & 0.69 & 4.79 & 0.01336 & 2.68 & MSGR & $\mathrm{E}$ & $\mathrm{D}$ & $24^{\circ} 27^{\prime} \mathrm{S}$ & (4) (PETRACCO, 2008) \\
\hline Hastula cinerea & 0.11 & 0.16 & 0.69 & 4.79 & 0.01336 & 2.68 & SF & $\mathrm{E}$ & $\mathrm{D}$ & $24^{\circ} 27^{\prime} \mathrm{S}$ & (4) (PETRACCO, 2008) \\
\hline \multicolumn{12}{|l|}{ Crustacea } \\
\hline \multicolumn{12}{|l|}{ Amphipoda } \\
\hline $\begin{array}{l}\text { Pseudorchestoidea } \\
\text { brasiliensis }\end{array}$ & 0.30 & 0.13 & 2.30 & 1.46 & 0.00223 & 2.08 & MSGR & E & $\mathrm{R}$ & $23^{\circ} 02^{\prime} \mathrm{S}$ & $\begin{array}{l}\text { (CARDOSO; VELOSO, } \\
\text { 1996) }\end{array}$ \\
\hline Pseudorchestoidea & & & & & & & & & & & (CARDOSO; VELOSO, \\
\hline brasiliensis & 0.28 & 0.13 & 2.16 & 1.46 & 0.00223 & 2.08 & SF & E & $\mathrm{R}$ & $23^{\circ} 02^{\prime} \mathrm{S}$ & 1996) \\
\hline $\begin{array}{l}\text { Pseudorchestoidea } \\
\text { brasiliensis }\end{array}$ & 028 & 013 & 221 & 098 & 000200 & 279 & MSGR & $\mathrm{E}$ & $\mathrm{R}$ & $23^{\circ} 00^{\prime} \mathrm{C}$ & (CARDOSO; VELOSO, \\
\hline $\begin{array}{l}\text { Drasillensls } \\
\text { Pseudorchestoidea }\end{array}$ & 0.28 & 0.13 & 2.21 & 0.98 & 0.00200 & 2.19 & MSGR & E & K & $2502 \mathrm{~S}$ & $\begin{array}{l}\text { (CARDOSO; VELOSO, } \\
\text { (CARDOSO V }\end{array}$ \\
\hline brasiliensis & 0.29 & 0.13 & 2.26 & 0.98 & 0.00200 & 2.79 & SF & E & $\mathrm{R}$ & $23^{\circ} 02^{\prime} \mathrm{S}$ & 1996) \\
\hline \multicolumn{12}{|l|}{ Isopoda } \\
\hline Excirolana braziliensis & 0.26 & 0.16 & 1.62 & 1.66 & 0.00249 & 2.93 & MSGR & $\mathrm{E}$ & $\mathrm{R}$ & $23^{\circ} 02^{\prime} \mathrm{S}$ & (VELOSO et al., 2003) \\
\hline Excirolana braziliensis & 0.32 & 0.16 & 1.98 & 1.66 & 0.00249 & 2.93 & SF & $\mathrm{E}$ & $\mathrm{R}$ & $23^{\circ} 02^{\prime} \mathrm{S}$ & (VELOSO et al., 2003) \\
\hline Excirolana braziliensis & 0.25 & 0.14 & 1.78 & 1.66 & 0.00201 & 2.74 & MSGR & $\mathrm{E}$ & $\mathrm{R}$ & $23^{\circ} 02^{\prime} \mathrm{S}$ & (VELOSO et al., 2003) \\
\hline Excirolana braziliensis & 0.26 & 0.14 & 1.84 & 1.66 & 0.00201 & 2.74 & SF & $\mathrm{E}$ & $\mathrm{R}$ & $23^{\circ} 02^{\prime} \mathrm{S}$ & (VELOSO et al., 2003) \\
\hline Excirolana braziliensis & 0.13 & 0.08 & 1.62 & 1.58 & 0.00144 & 3.44 & MSGR & $\mathrm{E}$ & $\mathrm{R}$ & $23^{\circ} 03^{\prime} \mathrm{S}$ & (CAETANO et al., 2006) \\
\hline Excirolana braziliensis & 0.56 & 0.25 & 2.24 & 1.65 & 0.00127 & 2.71 & MSGR & $\mathrm{E}$ & I & $23^{\circ} 03^{\prime} \mathrm{S}$ & (CAETANO et al., 2006) \\
\hline Excirolana armata & 0.45 & 0.13 & 3.55 & 0.89 & 0.00078 & 5.22 & MSGR & $\mathrm{E}$ & $\mathrm{D}$ & $24^{\circ} 27^{\prime} \mathrm{S}$ & $\begin{array}{l}\text { (PETRACCO et al., 2010, } \\
\text { 2012a) }\end{array}$ \\
\hline Excirolana armata & 0.47 & 0.13 & 3.77 & 0.89 & 0.00095 & 5.22 & SF & $\mathrm{E}$ & D & $24^{\circ} 27^{\prime} \mathrm{S}$ & $\begin{array}{l}\text { 2012a) } \\
\text { 201RACC et al., 2010, }\end{array}$ \\
\hline
\end{tabular}




\begin{tabular}{|c|c|c|c|c|c|c|c|c|c|c|c|}
\hline Group/Species & $\mathbf{P}$ & B & $\mathbf{P} / \mathbf{B}$ & LS & $\mathbf{M}_{\text {mean }}$ & $\mathbf{Z}$ & Met & DE & MS & Lat & Source \\
\hline Excirolana armata & 0.56 & 0.18 & 3.16 & 0.84 & 0.00078 & 6.91 & MSGR & $\mathrm{E}$ & $\mathrm{D}$ & $24^{\circ} 27^{\prime} \mathrm{S}$ & $\begin{array}{l}\text { (PETRACCO et al., 2010, } \\
\text { 2012a) } \\
\text { (PETRACCO et al., 2010, }\end{array}$ \\
\hline Excirolana armata & 0.59 & 0.18 & 3.32 & 0.84 & 0.00095 & 6.91 & $\mathrm{SF}$ & $\mathrm{E}$ & $\mathrm{D}$ & $24^{\circ} 27^{\prime} \mathrm{S}$ & 2012a) \\
\hline Excirolana armata & 0.35 & 0.19 & 1.86 & 1.55 & 0.00080 & 3.32 & MSGR & E & $\mathrm{D}$ & $25^{\circ} 20^{\prime} \mathrm{S}$ & ${ }^{(5)}($ SOUZA, 1998) \\
\hline Excirolana armata & 0.33 & 0.19 & 1.78 & 1.55 & 0.00080 & 3.32 & IS & $\mathrm{E}$ & $\mathrm{D}$ & $25^{\circ} 20^{\prime} \mathrm{S}$ & ${ }^{(5)}$ (SOUZA, 1998) \\
\hline Decapoda & & & & & & & & & & & (VELOSO; SALLORENZO, \\
\hline Emerita brasiliensis & 96.80 & 41.74 & 2.31 & 1.63 & 0.02047 & & MSGR & $\mathrm{E}$ & $\mathrm{R}$ & $22^{\circ} 56^{\prime} \mathrm{S}$ & $\begin{array}{l}\text { 2010) } \\
\text { (VELOSO; SALLORENZO, }\end{array}$ \\
\hline Emerita brasiliensis & 193.74 & 33.26 & 5.82 & 1.83 & 0.05569 & & MSGR & $\mathrm{E}$ & I & $22^{\circ} 57^{\prime} \mathrm{S}$ & 2010) \\
\hline Emerita brasiliensis & 39.86 & 4.91 & 8.12 & 0.73 & 0.00622 & 8.22 & MSGR & E & $\mathrm{R}$ & $23^{\circ} 02^{\prime} \mathrm{S}$ & (PETRACCO et al., 2003) \\
\hline Emerita brasiliensis & 46.88 & 4.91 & 9.55 & 0.73 & 0.00622 & 8.22 & SF & E & $\mathrm{R}$ & $23^{\circ} 02^{\prime} \mathrm{S}$ & (PETRACCO et al., 2003) \\
\hline Emerita brasiliensis & 150.95 & 23.09 & 6.54 & 0.66 & 0.01222 & 10.30 & MSGR & E & $\mathrm{R}$ & $23^{\circ} 02^{\prime} \mathrm{S}$ & (PETRACCO et al., 2003) \\
\hline Emerita brasiliensis & 156.07 & 23.09 & 6.77 & 0.66 & 0.01222 & 10.30 & SF & E & $\mathrm{R}$ & $23^{\circ} 02^{\prime} \mathrm{S}$ & (PETRACCO et al., 2003) \\
\hline Ocypode quadrata & 0.44 & 0.27 & 1.62 & 2.16 & 6.35439 & 3.84 & MSGR & $\mathrm{E}$ & $\mathrm{R}$ & $23^{\circ} 03^{\prime} \mathrm{S}$ & ${ }^{(6)}$ (GARCIA, 2003) \\
\hline Ocypode quadrata & 0.22 & 0.11 & 1.99 & 2.19 & 6.10005 & 2.39 & MSGR & E & $\mathrm{R}$ & $23^{\circ} 03^{\prime} \mathrm{S}$ & ${ }^{(6)}$ (GARCIA, 2003) \\
\hline Ocypode quadrata & 0.57 & 0.37 & 1.52 & 1.92 & 6.52680 & 2.95 & MSGR & E & I & $23^{\circ} 03^{\prime} \mathrm{S}$ & ${ }^{(6)}$ (GARCIA, 2003) \\
\hline Ocypode quadrata & 0.60 & 0.28 & 2.13 & 1.90 & 6.31353 & 3.88 & MSGR & $\mathrm{E}$ & I & $23^{\circ} 03^{\prime} \mathrm{S}$ & ${ }^{(6)}$ (GARCIA, 2003) \\
\hline $\begin{array}{l}\text { Austinixa } \\
\text { patagoniensis }\end{array}$ & 0.08 & 0.03 & 2.40 & 1.02 & 0.00445 & 3.43 & MSGR & $\mathrm{E}$ & $\mathrm{D}$ & $24^{\circ} 27^{\prime} \mathrm{S}$ & (4)(PETRACCO, 2008) \\
\hline $\begin{array}{l}\text { Austinixa } \\
\text { patagoniensis } \\
\text { Austinixa }\end{array}$ & 0.09 & 0.03 & 2.60 & 1.02 & 0.00445 & 3.43 & SF & E & D & $24^{\circ} 27^{\prime} \mathrm{S}$ & (4)(PETRACCO, 2008) \\
\hline $\begin{array}{l}\text { patagoniensis } \\
\text { Austinixa }\end{array}$ & 0.07 & 0.03 & 2.33 & 1.02 & 0.00448 & 3.43 & MSGR & $\mathrm{E}$ & $\mathrm{D}$ & $24^{\circ} 27^{\prime} \mathrm{S}$ & (4)(PETRACCO, 2008) \\
\hline patagoniensis & 0.08 & 0.03 & 2.49 & 1.02 & 0.00448 & 3.43 & SF & E & D & $24^{\circ} 27^{\prime} \mathrm{S}$ & (4)(PETRACCO, 2008) \\
\hline Callichirus major & 2.33 & 2.04 & 1.04 & 3.16 & 0.41359 & 2.57 & MSGR & E & $\mathrm{D}$ & $24^{\circ} 27^{\prime} \mathrm{S}$ & (4)(PETRACCO, 2008) \\
\hline Callichirus major & 6.35 & 6.00 & 1.06 & 3.00 & 0.41551 & 2.75 & MSGR & E & $\mathrm{D}$ & $25^{\circ} 20^{\prime} \mathrm{S}$ & (SOUZA et al., 1998) \\
\hline $\begin{array}{l}\text { Emerita brasiliensis } \\
\text { Polychaeta }\end{array}$ & 238.50 & 32.24 & 7.30 & 1.33 & 0.01402 & & IS & $\mathrm{E}$ & $\mathrm{D}$ & $32^{\circ} 20^{\prime} \mathrm{S}$ & (GIANUCA, 1985) \\
\hline Scolelepis chilensis & 1.92 & 0.97 & 1.97 & & 0.00026 & 2.96 & MSGR & $\mathrm{S}$ & & $23^{\circ} 49^{\prime} \mathrm{S}$ & ${ }^{(7)}$ (MACCORD, 2005) \\
\hline Scolelepis goodbodyi & 0.86 & 0.71 & 1.23 & & 0.00016 & 2.92 & MSGR & $S$ & & $23^{\circ} 49^{\prime} \mathrm{S}$ & ${ }^{(7)}(\mathrm{MACCORD}, 2005)$ \\
\hline Euzonus furciferus & 0.46 & 0.21 & 2.13 & 1.80 & 0.00086 & 3.20 & MSGR & E & D & $25^{\circ} 20^{\prime} \mathrm{S}$ & (SOUZA;BORZONE, 2007) \\
\hline Scolelepis squamata & 0.47 & 0.21 & 2.21 & 0.66 & 0.00018 & 2.52 & MSGR & $\mathrm{E}$ & $\mathrm{D}$ & $25^{\circ} 20^{\prime} \mathrm{S}$ & (SOUZA; BORZONE, 2000) \\
\hline Scolelepis squamata & 0.67 & 0.21 & 3.19 & 0.66 & 0.00018 & 2.52 & SF & E & $\mathrm{D}$ & $25^{\circ} 20^{\prime} \mathrm{S}$ & (SOUZA; BORZONE, 2000) \\
\hline Scolelepis squamata & 0.64 & 0.21 & 3.03 & 0.49 & 0.00018 & 5.52 & MSGR & E & D & $25^{\circ} 20^{\prime} \mathrm{S}$ & (SOUZA; BORZONE, 2000) \\
\hline $\begin{array}{l}\text { Scolelepis squamata } \\
\text { Thoracophelia }\end{array}$ & 0.50 & 0.21 & 2.38 & 0.49 & 0.00018 & 5.52 & SF & $\mathrm{E}$ & $\mathrm{D}$ & $25^{\circ} 20^{\prime} \mathrm{S}$ & (SOUZA; BORZONE, 2000) \\
\hline furcifera & 6.58 & 5.64 & 1.17 & 2.60 & 0.00346 & 3.80 & MSGR & $\mathrm{S}$ & & $27^{\circ} 34^{\prime} \mathrm{S}$ & (OTEGUI et al., 2012) \\
\hline Scolelepis gaucha & 1.84 & 0.14 & 12.92 & & 0.00005 & 11.35 & MSGR & $\mathrm{E}$ & D & $32^{\circ} 20^{\prime} \mathrm{S}$ & (SANTOS, 1994) \\
\hline
\end{tabular}

${ }^{(1)}$ CORTE G. N. Biologia populacional e reprodutiva de Anomalocardia brasiliana (Gmelin, 1791) (Bivalvia:Veneridae). 2011. Dissertação (Mestrado), UNICAMP, Campinas.

${ }^{(2)}$ SALVADOR L. B. Malacofauna da região entre-marés de praias de areia com fragmentos de rocha. 2001. Tese (Doutorado) UNESP, Rio Claro.

${ }^{(3)}$ YOKOYAMA L. Q. Nassarius vibex (Gastropoda, Nassariidae): crescimento e reprodução em bancos de mitilídeos de substrato areno-lamoso na costa sudeste do Brasil, 2010. Tese (Doutorado), UNICAMP, Campinas.

${ }^{(4)}$ PETRACCO M. Produção secundária da macrofauna bentônica da zona entremarés no segmento norte da praia do Una, litoral sul do estado de São Paulo, 2008. Tese (Doutorado), USP, São Paulo. 236p.

${ }^{(5)}$ SOUZA J. R. B. Produção secundária da macrofauna bentônica de praia de Atami - PR, 1998. Tese (Doutorado) UFPR, Curitiba.

${ }^{\left({ }^{6}\right)}$ GARCIA M. G. S. Comparação da biologia do caranguejo Ocypode quadrata (Fabricius, 1787) (Crustacea, Decapoda, Ocypodidae) em duas praias arenosas expostas do litoral do Rio de Janeiro, RJ. 2003. Dissertação (Bacharelado), UNIRIO, Rio de Janeiro.

${ }^{7)}$ MACCORD F. S. Dinâmica populacional e biologia reprodutiva de duas espécies de Scolelepis (Spionidae) e de Laeonereis acuta (Nereididae) (Annelida:Polychaeta). 2005. Tese (Doutorado), USP, São Paulo. 130p.

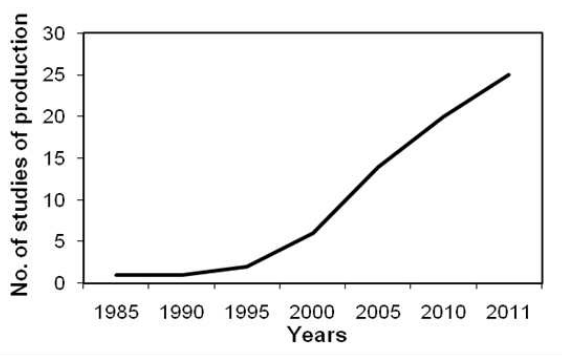

Fig. 1.Cumulative number of studies on production of Brazilian sandy beach macrofauna over the years. 

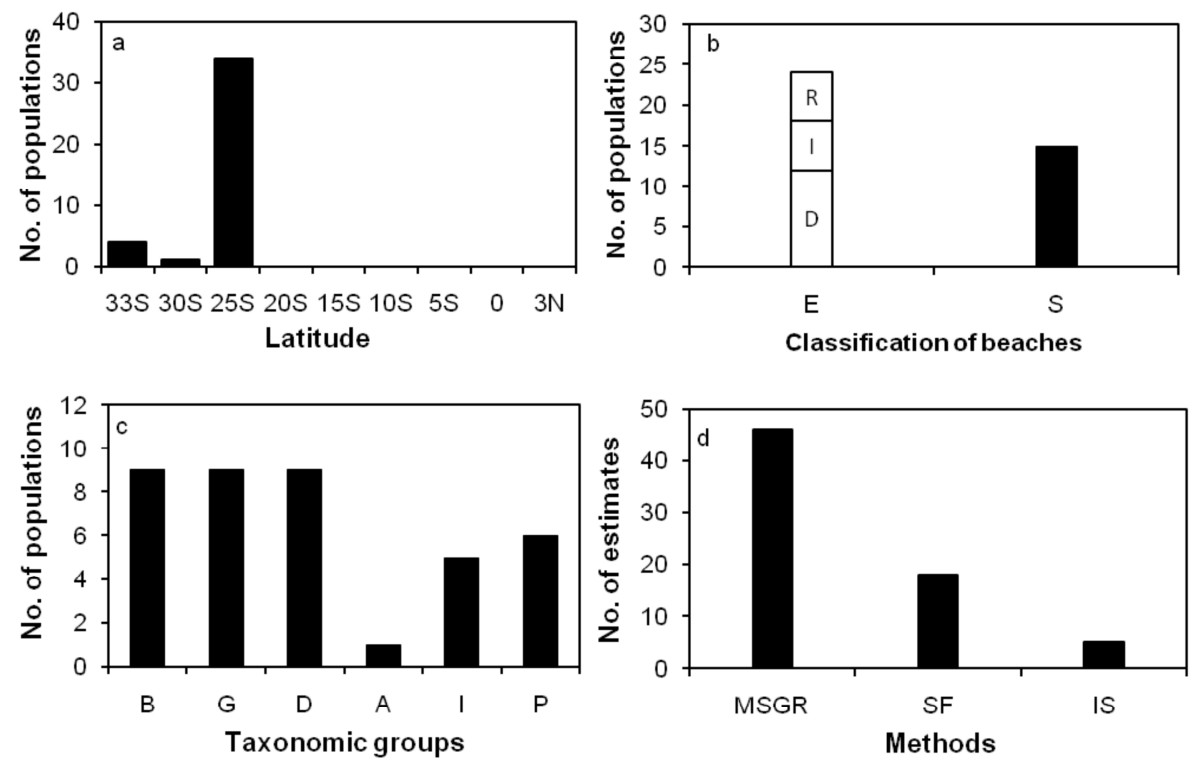

Fig. 2. Distribution of macrofauna populations studied in Brazilian sandy beaches for which the production was estimated according to: (a) latitude, (b) beach classification according to degree of exposure of sandy beaches (exposed: E and sheltered: S), and morphodynamic state of exposed sandy beaches (dissipative: D, intermediate: I, and reflective: R), (c) taxonomic groups (Bivalvia: B, Gastropoda: G, Decapoda, D, Amphipoda, A, Isopoda, I, and Polychaete, P) and (d) employed methods (mass-specific growth rate: MSGR, size-frequency: SF and increment summation method, IS).

The annual production of the macrofauna has mostly been obtained with size-based methods (64), with a predominance of the mass-specific growth rate method (MSGR, 46) in relation to the frequencysize method (SF, 18). Cohort methods have been represented only by the increment summation (IS, 5) (Fig. 2d and Table 1). For $40 \%$ of the populations studied, two methods of production have been employed and, generally, the size-frequency method has been used simultaneously with the MSGR method. The predominance of size-based methods in studies on Brazilian sandy beach macrofauna was to be expected since, in subtropical regions, cohorts cannot generally be followed and, consequently, cohort methods cannot be employed. In fact, among the five estimates obtained with cohort methods, three were obtained for temperate beaches in southern Brazil (GIANUCA, 1985). The predominance of the mass-specific growth rate method (MSGR) in relation to the size-frequency method was also expected, since the former is considered more accurate than the latter (BREY, 2001) and the calculation of the production by the MSGR is performed directly from the parameters of the von Bertalanffy growth function $\left(\mathrm{K}\right.$ and $\left.\mathrm{L}_{\infty}\right)$, which have almost always obtained in production studies.

\section{Trends in Production and P/B Ratio of the More} Representative Taxonomic Groups and Species

Bivalves and, mainly, decapods have shown high median and mean values of production and biomass (Table 2). Conversely, the $\mathrm{P} / \mathrm{B}$ ratio and life span of gastropods have been, respectively, low and long. Decapods and gastropods have presented high values of mean body mass. These general trends in production and in $\mathrm{P} / \mathrm{B}$ ratio among the taxonomic groups followed a pattern similar to that observed for a broader analysis performed for sandy beach macrofauna around the world (PETRACCO et al., unpublished data). The high values of decapods and bivalve production have been due to high mean annual biomass (Table 2). Similarly, the low production of isopods can be attributed to this group's low biomass. Low values of P/B ratio for gastropods have been due to the slow growth and long life span of the species of this group. Conversely, the high $\mathrm{P} / \mathrm{B}$ ratio of decapods has mainly been due to the presence of several populations of the mole crab Emerita brasiliensis in the dataset (see below). 
Table 2. Median (med) and mean ( \pm SE) of annual production $\left(\mathrm{P}: \mathrm{g} \mathrm{AFDM} \mathrm{m}^{-2} \mathrm{yr}^{-1}\right)$, mean annual biomass $\left(\mathrm{B}: \mathrm{g} \mathrm{AFDM} \mathrm{m}^{-2}\right)$, annual $\mathrm{P} / \mathrm{B}$ ratio $\left(\mathrm{yr}^{-1}\right)$, life span (LS: years) and mean body mass $\left(\mathrm{M}_{\text {mean }}\right.$ : $\mathrm{g}$ AFDM) for the taxonomic groups, Bivalvia (B), Gastropoda (G), Isopoda (I), Decapoda (D) and Polychaeta (P).

\begin{tabular}{|c|c|c|c|c|c|c|c|c|c|c|c|c|c|c|c|}
\hline Group & & P & & & B & & & $\mathrm{P} / \mathrm{B}$ & & & LS & & & $\mathrm{M}_{\text {mean }}$ & \\
\hline & $\mathrm{n}$ & mean & med & $\mathrm{n}$ & mean & med & $\mathrm{n}$ & mean & med & $\mathrm{n}$ & mean & med & $\mathrm{n}$ & mean & med \\
\hline B & 10 & $\begin{array}{l}22.32 \pm \\
18.17\end{array}$ & 2.57 & 10 & $\begin{array}{l}12.66 \pm \\
10.53\end{array}$ & 1.38 & 10 & $\begin{array}{l}1.91 \pm \\
0.27\end{array}$ & 1.76 & 9 & $\begin{array}{l}2.58 \pm \\
0.41\end{array}$ & 2.00 & 7 & $\begin{array}{l}0.021 \pm \\
0.007\end{array}$ & 0.002 \\
\hline G & 11 & $\begin{array}{l}1.01 \pm \\
0.47\end{array}$ & 0.19 & 11 & $\begin{array}{l}1.30 \pm \\
0.75\end{array}$ & 0.17 & 11 & $\begin{array}{l}1.27 \pm \\
0.28\end{array}$ & 1.16 & 11 & $\begin{array}{l}3.67 \pm \\
0.34\end{array}$ & 4.00 & 11 & $\begin{array}{l}0.198 \pm \\
0.123\end{array}$ & 0.020 \\
\hline I & 7 & $\begin{array}{l}0.37 \pm \\
0.06\end{array}$ & 0.34 & 7 & $\begin{array}{l}0.16 \pm \\
0.02\end{array}$ & 0.16 & 7 & $\begin{array}{l}2.31 \pm \\
0.31\end{array}$ & 1.82 & 7 & $\begin{array}{l}1.40 \pm \\
0.14\end{array}$ & 1.58 & 7 & $\begin{array}{l}0.001 \pm \\
0.000\end{array}$ & 0.001 \\
\hline D & 11 & $\begin{array}{l}66.88 \pm \\
26.98\end{array}$ & 6.35 & 11 & $\begin{array}{l}13.08 \pm \\
4.86\end{array}$ & 4.91 & 11 & $\begin{array}{l}3.78 \pm \\
0.85\end{array}$ & 2.41 & 11 & $\begin{array}{l}1.68 \pm \\
0.26\end{array}$ & 1.63 & 11 & $\begin{array}{l}1.236 \pm \\
0.760\end{array}$ & 0.021 \\
\hline P & 7 & $\begin{array}{l}1.83 \pm \\
0.82\end{array}$ & 0.86 & 7 & $\begin{array}{l}1.16 \pm \\
0.76\end{array}$ & 0.21 & 7 & $\begin{array}{l}3.55 \pm \\
1.58\end{array}$ & 2.13 & 4 & $\begin{array}{l}1.61 \pm \\
0.69\end{array}$ & 1.23 & 7 & $\begin{array}{l}0.001 \pm \\
0.000\end{array}$ & 0.000 \\
\hline
\end{tabular}

Brazilian studies have referred to bivalve populations living in exposed and sheltered sandy beaches. Anomalocardia brasiliana, the most frequently studied species, inhabits sheltered sandy beaches and has been heavily exploited along the coast (e.g. MATTOS; CARDOSO, 2012). Populations of this species can attain considerable productions (5.45 and $11.60 \mathrm{~g} \mathrm{AFDM} \mathrm{m}^{-2} \mathrm{yr}^{-1}$ ) (Table 1). Other species of bivalves harvested by man, Donax hanleyanus and Mesodesma mactroides, have high values of production and $\mathrm{P} / \mathrm{B}$ on the dissipative Cassino beach, in Rio Grande do Sul state, as a result of the high primary production on this beach (GIANUCA, 1985). On the other hand, most gastropod species in the dataset inhabit sheltered sandy beaches and have low $\mathrm{P} / \mathrm{B}$ ratio, i.e., they replace their biomass slowly. Consequently, these species probably have little resilience to environmental disturbances (sensu Tumbiolo and Downing, 1994).

Among the decapods, the production and productivity of the filter-feeder Emerita brasiliensis has mainly been addressed for the populations in Rio de Janeiro, with high values on dissipative, intermediate and reflective beaches (PETRACCO et al., 2003; VELOSO; SALLORENZO, 2010). Production is the product of mass-specific growth rate $(\mathrm{G})$ and biomass. As these factors are often negatively correlated, both parameters are rarely maximized simultaneously (HURYN; WALLACE, 2000). However, the high values of production of this species are a result of high mass-specific growth rate deriving from the high parameter of curvature of the von Bertalanffy growth function (see VELOSO; CARDOSO, 1999; PETRACCO et al., 2003; VELOSO; SALLORENZO, 2010) and high biomass. Moreover, the high P/B ratios of this filter-feeder are corroborated by their low life span (Table 1). The high motility of some macrobenthos groups (e.g. amphipods) can enhance their metabolism and, consequently, the $\mathrm{P} / \mathrm{B}$ ratios (CUSSON; BOURGET, 2005). This condition seems to apply to E. brasiliensis, which shows high motility to enable it to survive in the harsher swash zone of exposed beaches with different morphodynamic states. Consequently, unlike the well-documented negative relationship between $\mathrm{P} / \mathrm{B}$ and mean body mass, $E$. brasiliensis shows higher $\mathrm{P} / \mathrm{B}$ ratios than most species with lower mean body mass, such as isopods and polychaetes (Table 1). Furthermore, E. brasiliensis is used as a food resource by fish and crab species (McLACHLAN; BROWN, 2006) and is, thus, an important component in the energy flow of sandy beach ecosystems. Another decapod which can attain considerable production is the thalassinid Callichirus major, which is often exploited as fishing bait (RODRIGUES; SHIMIZU, 1997; SOUZA; BORZONE, 2003; BOTTER-CARVALHO et al., 2007). However, in contrast with $E$. brasiliensis, $C$. major shows a low $\mathrm{P} / \mathrm{B}$ ratio, due to its slow growth, long life span ( 3 yrs: Table 1$)$ and high mean body mass (SOUZA et al., 1998). Considering that species with a high $\mathrm{P} / \mathrm{B}$ ratio generally offer a higher maximum sustainable yield, the low $\mathrm{P} / \mathrm{B}$ ratio indicates that $C$. major is more susceptible to harvesting (sensu BREY, 1990).

The high number of estimates for the peracarid crustaceans of the genus Excirolana on exposed beaches in southern and southeastern Brazil results from the fact that two species of this genus are frequent and abundant on Brazilian beaches (CAETANO et al., 2006; PETRACCO et al., 2010). However, unlike Emerita brasiliensis, low production and $\mathrm{P} / \mathrm{B}$ ratios have been estimated for the scavenger Excirolana armata, and mainly for the detritivorous $E$. braziliensis. Some conservative $\mathrm{P} / \mathrm{B}$ ratios for these species are at variance with some of their life history traits such as high motility, low mean body mass and short life span, and may be due to an inadequate sampling regime and/or sampling selectivity (PETRACCO et al., 2012a).

The production of the polychaetes of the genus Scolelepis has been studied on exposed and sheltered dissipative beaches of the southeastern and southern Brazilian coast (SANTOS, 1994; SOUZA; BORZONE, 2000). Generally, Scolelepis populations have low production $\left(\sim 0.5 \mathrm{~g} \mathrm{AFDM} \mathrm{m}^{-2} \mathrm{yr}^{-1}\right)$ and $\mathrm{P} / \mathrm{B}$ 
ratios around $2 \mathrm{yr}^{-1}$, despite the very high productivity of Scolelepis gaucha on Cassino beach (12.92 $\mathrm{yr}^{-1}$ : SANTOS, 1994). The deposit feeder Thoracophelia furcifera has been studied on exposed and sheltered sandy beaches of southern Brazil (SOUZA; BORZONE, 2007; OTEGUI et al. 2012). The considerable production of this species on a sheltered beach (6.6 $\mathrm{g} \mathrm{AFDM} \mathrm{m}^{-2} \mathrm{yr}^{-1}$ : OTEGUI et al., 2012), higher than that on an exposed beach (0.46 g AFDM $\mathrm{m}^{-2} \mathrm{yr}^{-1}$ : SOUZA; BORZONE, 2007) is related to the fact that the latter is a probably a more favorable environment for the deposit-feeder polychaete.

The production ratio over some period (often a year) for the mean biomass over that same period $(\mathrm{P} / \mathrm{B}$ ratio) is of major interest, both practical and theoretical, in studies on macrobenthos secondary production. In practical terms, if the biomass is known, then, the $\mathrm{P} / \mathrm{B}$ ratio can be used to estimate production. Within a theoretical perspective, it is interesting to know how the $\mathrm{P} / \mathrm{B}$ ratio varies among taxa and in accordance with environmental conditions in order to develop predictive energetic models (STITES; BENKE, 1989). Furthermore, population production is often expressed as $\mathrm{P} / \mathrm{B}$ ratio, in the hope of yielding standardized production values comparable with those of species or populations with differing biomasses (PLANTE; DOWNING, 1989). Thus, accurate estimates of $\mathrm{P} / \mathrm{B}$ ratio must be obtained in studies on secondary production. Since the accuracy of production and, particularly, the $\mathrm{P} / \mathrm{B}$ ratio depends on an accurate representation of the density and size structure of a population (BENKE; HURYN, 2006), sample selectivity is a determining factor in obtaining reliable estimates of production and $\mathrm{P} / \mathrm{B}$ ratios. Higher values of the instantaneous mortality rate $(\mathrm{Z})$ compared to the respective $\mathrm{P} / \mathrm{B}$ ratios may indicate selectivity against smaller individuals in the population (BREY, pers. comm.). The $\mathrm{Z}$ estimated for sandy beach macrofauna was significantly higher than the respective $\mathrm{P} / \mathrm{B}$ ratios (paired $t$-test: $\mathrm{t}_{1,39}=7.06$, $\mathrm{p}<0.0001)$. Moreover, the relationship between these two variables, described by a significant linear model, shows a significantly gentler slope than that of Allen's relationship $(\mathrm{Z}=\mathrm{P} / \mathrm{B}$, slope $=1) \quad\left(t_{1,76}=2.11, \quad \mathrm{p}<0.05\right)$ (Fig. 3). Several populations, recorded in table 1, show evidence of sampling selectivity against smaller individuals. For instance, this fact can be observed in species such as the gastropod Olivancillaria vesica (CAETANO et al., 2003), and the bivalve Tivela mactroides (TURRA et al., unpublished data) that have sharp size-stratified distribution, with the smallest individuals inhabiting the subtidal zone. Similarly, the decapod Callichirus major has a size structure from which smaller individuals are absent due to the impossibility of collecting the newly recruited individuals. According to Shimizu and Rodrigues (1999), the recruitment of C. major is detected only 5 to 7 months after the estimated post larvae settlement period due to the selectivity of the sampling method (yabby-pump). In addition, the recruits in some species can escape the frequently used $0.5-\mathrm{mm}$ mesh, as suggested for Scolelepis squamata and Excirolana species (SOUZA; BORZONE, 2000; PETRACCO et al., 2012a). On the other hand, for populations which show sampling selectivity against the larger individuals, the $\mathrm{P} / \mathrm{B}$ ratio is overestimated, as suggested by Denadai et al. (2004) for the gastropod Cerithium atratum. Consequently, sampling selectivity should be given greater importance in studies on secondary production of sandy beach macrofauna, especially for species that are not restricted to the intertidal zone and supralittoral.

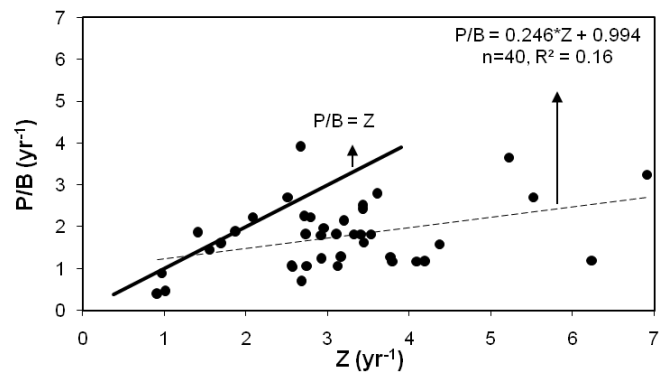

Fig. 3. P/B ratios plotted against the instantaneous mortality rate $(\mathrm{Z})$ (dashed line) obtained for Brazilian sandy beach macrofauna. Allen's relationship: $\mathrm{P} / \mathrm{B}=\mathrm{Z}$ (full line).

\section{Approaches of the Studies and Guidelines for Future Research}

The most common study approach to sandy beach macrofauna focuses on the production analyses and especially on the $\mathrm{P} / \mathrm{B}$ ratio, according to the life history traits of a single or a few populations, and on the comparison of the species studied to other populations/species of the same taxonomic group (CARDOSO; VELOSO, 1996; SOUZA; BORZONE, 2000; CAETANO et al., 2003; DENADAI et al., 2004, among others). For these comparisons, wellestablished relationships between $\mathrm{P} / \mathrm{B}$ and life history traits such as life span, mean body mass, population size structure, and mortality, are used (sensu ALLEN, 1971; ROBERTSON, 1979; BREY, 1990; see PETRACCO et al., 2012b). These studies are important because: i) they give the production and productivity levels in populations of different representative species of sandy beaches, ii) they contribute to the understanding of the variation in production especially of productivity with reference to life history attributes, and iii) life history-production studies have served an important basis for the development of empirical methods (BENKE; 
HURYN, 2010). Therefore, studies using this approach should be undertaken on all the major taxonomic groups of Brazilian sandy beach macrofauna, especially for amphipods and polychaetes whose production is very poorly known.

One of the main objectives regarding ecological patterns on sandy beaches in the last decade has related to the response of several life history traits either to contrasting morphodynamic states of beaches or to latitudinal variation (CARDOSO; DEFEO, 2003, 2004; DEFEO; CARDOSO, 2002, 2004; DEFEO; McLACHLAN, 2005; PETRACCO et al., 2010). In the first case, an interesting study was conducted by Santos (1994), who analyzed the production and P/B ratio of Scolelepis gaucha according to the variation of morphodynamic state (beach slope) along Cassino beach. More recently, some studies have compared the production and $\mathrm{P} / \mathrm{B}$ ratios of populations of the same species living on beaches with different environmental characteristics (e.g. CAETANO et al., 2006; VELOSO; SALLORENZO, 2010). The elucidation of how the production and $\mathrm{P} / \mathrm{B}$ ratio of species of different taxonomic and functional groups and of intertidal and supralittoral forms respond to different environmental beach conditions is an essential step to establishing production patterns in these habitats. This is clearly a topic of increasing interest in the ecology of sandy beaches. Likewise, establishing the latitudinal variation of the $\mathrm{P} / \mathrm{B}$ ratio, a powerful variable which integrates the dynamic relationship between growth rate, biomass, and production (HURYN; BENKE, 2007), is very important within a macroecological perspective (e.g. CARDOSO; VELOSO, 2003; PETRACCO et al., 2012a).Therefore, production studies on the North and Northeastern Brazilian coast would be of great importance, as they would allow the establishment of relationships between production and productivity and temperature and latitude.

Unlike what has been observed for marine ecosystems and, particularly, for sandy beaches, there are many annual production estimates for entire communities of freshwater macroinvertebrates, and production has been used as an essential variable in several ecologic approaches (BENKE, 1993; BENKE; HURYN, 2006; BENKE; HURYN, 2010 and references therein). These approaches include: i) comparison between production and $\mathrm{P} / \mathrm{B}$ ratio of taxonomic and trophic groups, ii) food web analysis, ii) response of different species' production and $\mathrm{P} / \mathrm{B}$ ratios to different human-induced disturbances, iii) development of predictive models to estimate the production and $\mathrm{P} / \mathrm{B}$ ratios, iv) niche overlap and interspecific competition, and v) importance of meiofauna versus macrofauna, among others (BENKE; HURYN, 2006; BENKE, 2010; BENKE; HURYN, 2010). Some of these approaches could be used in studies on the production of sandy beach macrofauna. For instance, no study has so far used production to assess the effects of the several humaninduced disturbances which frequently affect the Brazilian sandy beach macrofauna. Production and $\mathrm{P} / \mathrm{B}$ ratio could be employed together with abundance and biomass to evaluate the effects of these disturbances since production is a composite measure of density, biomass, growth rate, reproduction and survivorship (BENKE, 1993), which are frequently modified due to different human-induced disturbances (e.g. LOZOYA; DEFEO, 2006). Moreover, the production response might be different from the biomass response and offer a more complete picture of the disturbance and recovery of macrofauna than abundance and diversity (sensu Benke, 2010). On the other hand, considering the drastic changes that sandy beaches might suffer due to global climate change (BROWN; McLACHLAN, 2002), studies on the monitoring of the sandy beach macrofauna are critical to provide support for the management of this important group. In Brazil, the ReBentos network, recently created, has brought together various researchers who undertake research into marine benthic organisms, and aims at understanding and predicting the effects of climate change on the benthos of intertidal and shallow sublitoral regions of the Brazilian coast. Production data can, therefore, be a useful tool for the monitoring of Brazilian sandy beach macrofauna.

Another important ecological issue addressed recently relating to sandy beach macrofauna is interspecific competition. Some studies have observed negative correlations between abundance of species and the decrease of growth rate due to competition (e.g. DEFEO et al., 1997; CARDOSO; VELOSO, 2003; DEFEO; McLACHLAN, 2005; HERRMANN et al., 2009). Since production and P/B ratio are related to these two variables, production could be used in studies regarding interspecific competition. On the other hand, the importance of the meiofauna compared to the macrofauna is an essential issue regarding sandy beaches and production seems to be more appropriate than biomass to address this issue. Thus, production estimates could be used to evaluate several issues beyond the relationship between life history characteristics and production and productivity. Moreover, despite the fact that the uncertain quantification of production estimates is a long-standing problem, bootstrapping has been used to estimate confidence intervals for the production estimates for populations and communities, calculated by both size-frequency and instantaneous growth methods (see BENKE; HURYN, 2006 and references therein).

In summary, despite the recent increase in production studies, the lack of estimates on beaches 
located on the northern and northeastern Brazilian coast, as well as the scarcity of estimates for polychaetes and amphipods are important gaps in studies on Brazilian sandy beach macrofauna. For the attainment of reliable production estimates, efforts must focus on adequately sampling the populations, especially those whose distribution extends to the infralittoral. In addition to life history-production studies, other approaches, such as the effects of the disturbances caused by humans and related to different morphodynamic states on production must be addressed.

\section{ACKNOWLEDGEMENTS}

This paper is part of the $\mathrm{PhD}$ thesis and Postdoctorate of M. Petracco at the Instituto Oceanográfico da Universidade de São Paulo (IOUSP). The authors wish to thank several colleagues who provided information from their studies and thus contributed to the development of this study. Thanks are due to D.T. Ramos for the English revision. We are also grateful to the one anonymous reviewer whose suggestions enriched the text. This study was supported by FAPESP (Process no. 2008/11053-0) and CNPq (Process no. 163181/2011$0)$.

\section{REFERENCES}

ALLEN, K. R. Relation between production and biomass. J. Fish. Res. Bd. Can., v. 28, p. 1573-1581, 1971.

AMARAL, A. C. A.; DENADAI, M. R.; TURRA, A.; RIZZO A. E. Intertidal macrofauna in Brazilian subtropical tide-dominated sandy beaches. J. coast. Res., v. 35(SI), p. 446-455, 2003.

BENKE, A. C. Secondary production of aquatic insects. In: RESH, V. H.; ROSENBERG, D. M. (Ed.). Ecology of aquatic insects. New York: Praeger Scientific, 1984. p. 289-322.

BENKE, A. C. Concepts and patterns of invertebrate production in running waters. Verh. Internate. Verein. Limnol., v. 25, p. 15-28, 1993.

BENKE A. C. Secondary production as part of bioenergetic theory-contributions from freshwater benthic science. River Res. Applic., v. 26, p. 36-44, 2010.

BENKE, A. C.; HURYN, A. D. Secondary production of macroinvertebrates. In: HAUER F. T.; LAMBERTI G. A. (Ed.). Methods in stream ecology. $2^{\text {nd }}$ ed. Massachussets: Academic Press, 2006. p. 691-710.

BENKE, A. C.; HURYN, A. D. Benthic invertebrate production-facilitating answers to ecological riddles in freshwater ecosystems. J. N. Am. Benthol. Soc., v. 29, p. 264-285, 2010.

BOTTER-CARVALHO, M. L.; SANTOS, P. J. P.; CARVALHO, P. V. V. C. Population dynamics of Callichirus major (Say, 1818) (Crustacea, Thalassinidea) on a beach in northeastern Brazil. Estuar. Coast. Shelf Sci., v. 71, 508-516, 2007.
BREY, T. Estimating productivity of macrobenthic invertebrates from biomass and mean individual weight. Meeresforsch., v. 32, p. 329-34, 1990.

BREY, T. Population dynamics in benthic invertebrates.A virtual handbook.Version 1.2. Germany: Alfred Wegener Institute for Polar and Marine Research, <http://www.thomas-brey.de/science/virtualhandbook, 2001>.

BREY, T.; RUMOHR, H.; ANKAR, S. Energy content of macrobenthic invertebrates: general conversion factors from weight to energy. J. Exp. Mar. Biol. Ecol., v. 117, p. 271-278, 1988.

BROWN, A. C.; McLACHLAN, A. Sandy shore ecosystems and the threats facing them: some predictions for the year 2025. Environ. Conserv., v. 29, p. 62-77, 2002.

CABRINI, T. M. B.; CARDOSO, R. S. Produção secundária dos gastrópodes Cerithium atratum e Nassarius vibex, na baía de Sepetiba, Rio de Janeiro. In: CONGRESSO BRASILEIRO DE OCEANOGRAFIA, IV. Natal, Rio Grande do Norte, 2010.

CABRINI, T. M. B.; CARDOSO, R. S. Population biology of Nassarius vibex (Say, 1822) on a sheltered beach in Southeastern Brazil. J. shellfish Res., v.31, p. 808-815, 2012.

CAETANO, C. H. S.; CARDOSO, R. S.; VElOSO V. G.; SILVA E. S. Population biology and secondary production of Excirolana braziliensis (Isopoda: Cirolanidae) in two sandy beaches of southeastern Brazil. J. coast. Res, v. 22, p. 825-835, 2006.

CAETANO, C. H. S.; VElOSO, V. G.; CARDOSO, R. S. Population biology and secondary production of Olivancillaria vesica vesica (Gmelin, 1791) (Gastropoda: Olividae) on a sandy beach in southeastern Brazil. J. Mollus. Stud., v. 69, p. 67-73, 2003.

CARDOSO, R. S.; DEFEO, O. Geographical patterns in reproductive biology of the Pan-American sandy beach isopod Excirolana braziliensis. Mar. Biol., v. 143, p. 573-581, 2003.

CARDOSO, R. S.; DEFEO, O. Biogeographical patterns in life history traits of the Pan-American sandy beach isopod Excirolana braziliensis. Estuar. Coast. Shelf Sci., v. 61, p. 559-568, 2004.

CARDOSO, R. S.; VELOSO, V. G. Population biology and secondary production of the sandhopper Pseudorchestoidea brasiliensis (Amphipoda:Talitridae) at Prainha Beach, Brazil. Mar. Ecol. Prog. Ser., v. 142, p. 111-119, 1996.

CARDOSO, R. S.; VELOSO, V. G. Population dynamics and secondary production of the wedge clam Donax hanleyanus on a high energy, subtropical beach of Brazil. Mar. Biol., v. 142, p. 153-162, 2003.

CUSSON, M.; BOURGET, E. Global patterns of macroinvertebrate production in marine benthic habitats. Mar. Ecol. Prog. Ser., v. 297, p. 1-14, 2005.

DEFEO, O.; BRAZEIRO, A.; DE ALAVA, A.; RIESTRA, G. Is sandy beach macroinfauna only physically controlled? Role of substrate and competition in isopods. Estuar. Coast. Shelf Sci., v. 45, p. 453-462, 1997.

DEFEO, O.; CARDOSO, R. S. Macroecology of population dynamics and life history traits of the mole crab Emerita brasiliensis in Atlantic sandy beaches of South America. Mar. Ecol. Prog. Ser., v. 239, p. 169-179, 2002. 
DEFEO, O.; CARDOSO, R. S. Latitudinal patterns in abundance and life-history traits of the mole crab Emerita brasiliensis on South American sandy beaches. Divers. Distrib., v. 10, p. 89-98, 2004.

DEFEO, O.; McLACHLAN, A. Patterns, processes and regulatory mechanisms in sandy beach macrofauna: a multi-scale analysis. Mar. Ecol. Prog. Ser., v. 295, p. 1$20,2005$.

DEFEO, O.; McLACHLAN, A.; SCHOEMAN, D. S ; SCHLACHER, T. A.; DUGAN, J.; JONES, A.; LASTRA, M.; SCAPINI, F. Threats to sandy beaches ecosystems: a review. Estuar. Coast. Shelf Sci., v. 81, p. 1-12, 2009.

DENADAI, M. R.; AMARAL, A. C. Z.; TURRA, A Biology of a tropical intertidal population of Cerithium atratum (Born, 1778) (Mollusca, Gastropoda). J. Nat. Hist., v. 38, p. 1695-1710, 2004.

DEXTER, D. M. Sandy beach community structure: the role of exposure and latitude. J. Biogeogr., v. 19, p. 59-66, 1992.

DOWNING, J. A. Assessment of secondary production: the first step. In: DOWNING J. A.; RIGLER F. H. (Ed.). A manual on the assessment of secondary productivity in fresh waters. Oxford: Blackwell Scientific Publications, 1984. p. 1-18. (IBP Handbook no.17).

DUGAN, J. E.; HUBBARD, D. M.; RODIL, I. F.; REVELL, D. L.; SCHROETER, S. Ecological effects of coastal armoring on sandy beaches. Mar. Ecol., v. 29, p. 160170, 2008

GIANUCA, N. M. Ecology of a sandy beach in Southern Brazil. 1985. PhD thesis, University of Southampton.

HERRMANN, M.; CARSTENSEN, D.; FISHER, S.; LAUDIEN, J.; PENCHASZADEH, P. E.; ARNTZ, W. E. Population structure, growth, and production of the wedge clam Donax hanleyanus (Bivalvia: Donacidae) from Northern Argentinean beaches. J. shellfish Res., v. 28, p. 511-526, 2009.

HURYN, A. D.; BENKE, A. C. Relationship between biomass turnover and body size for stream communities. In: HILDREW, A.G.; RAFFAELLI, D. G.; EDMONDSBROWN, R. (Ed.). Body size: the structure and function on aquatic ecosystems. Cambridge, UK: Cambridge University Press, 2007. p 55-76.

HURYN, A. D.; WALLACE, J. B. Life history and production of stream insects. Annu. Rev. Entomol., v. 45 , p. 83-110, 2000.

LAUDIEN, J.; BREY, T.; ARNTZ, W. E. Population structure, growth and production of the surf clam Donax serra (Bivalvia, Donacidae) on two Namibian sandy beaches. Estuar. Coast. Shelf Sci., v. 58S, p. 105-115, 2003.

LOZOYA, J. P.; DEFEO, O. Effects of a freshwater canal discharge on an ovoviviparous isopod inhabiting an exposed sandy beach. Mar. Freshw. Res., v. 57, p. 421428, 2006

MATTOS, G.; CARDOSO R. S. Population dynamics of two suspension-feeding bivalves on a sheltered beach in southeastern Brazil. Helgol. Mar. Res., v. 66, p. 393 400, 2012.

McLACHLAN, A. The definition of sand beaches in relation to exposure: a simple rating system. S. Afr. J. Sci., v. 76, p. $137-138,1980$.
McLACHLAN, A.; BROWN, A. Sandy beaches as ecosystems. Amsterdam: Elsevier Science Publishers, 2006. 373p

McLACHLAN, A.; DUGAN J. E.; DEFEO O.; ANSELL, A. D.; HUBBARD, D. M.; JARAMILLO E.; PENCHASZADEH, P. Beach clam fisheries. Oceanogr. Mar. Biol., v. 34, p. 163-232, 1996.

OTEGUi, M. B. P.; BLANKESTEYN, A.; PAGLIOSA, P. R. Population structure, growth and production of Thoracophelia furcifera (Polychaeta:Opheliidae) on a sandy beach in Southern Brazil. Helgol. Mar. Res., v. 66, p. 479-488, 2012.

PETERSON, C. H.; SUMMERSON, H. C.; THOMSON, E.; LENIHAN, H. S.; GRABOWSKI, J.; MANNING L.; MICHELI F.; JOHNSON G. Synthesis of linkages between benthic and fish communities as a key to protecting essential fish habitat. Bull. Mar. Sci., v.66, p. 759-774, 2000

PETRACCO, M.; CARDOSO, R. S.; CORBISIER, T. N. Population biology of Excirolana armata (Dana, 1853) (Isopoda, Cirolanidae) on an exposed sandy beach in Southeastern Brazil. Mar. Ecol., v. 31, p. 330-340, 2010.

PETRACCO, M.; CARDOSO, R. S.; CORBISIER T. N.; TURRA A. Secondary production of sandy beach macrofauna: An evaluation of predictive models. Estuar. Coast. Shelf Sci., v.115, p. 359-365, 2012 b.

PETRACCO, M.; CARDOSO, R. S.; TURRA A.; CORBISIER T. N. Production of Excirolana armata (Dana, 1853) (Isopoda, Cirolanidae) on an exposed sandy beach in Southeastern Brazil. Helgol. Mar. Res., v. 66 , p. $265-274,2012$ a.

PETRACCO, M.; VELOSO, V. G.; CARDOSO, R. S. Population dynamics and secondary production of Emerita brasiliensis (Crustacea: Hippidae) at Prainha Beach, Brazil. Mar. Ecol., v. 24, p. 385-391, 2003.

PLANTE C.; DOWNING, J. A. Production of freshwater invertebrate populations in lakes. Can. J. Fish. Aquat. Sci., v. 46, p. 1489-1498, 1989.

RICCIARDI, A.; BOURGET, E. Weight-to-weight conversion factors for marine benthic macroinvertebrates. Mar. Ecol. Prog. Ser., v. 163, p. 245-251, 1998.

ROBERTSON, A. I. The relationship between annual production:biomass ratio and lifespan for marine macrobenthos. Oecologia, v. 38 p. 193-202, 1979.

RODRIGUES, S. A.; SHIMIZU, R. M. Autoecologia de Callichirus major (Say, 1818). Oecol. Bras., v. 3, p. 155-177, 1997.

SARDÁ, R.; PINEDO, S.; DUESO, A. Estimating secondary production in natural populations of polychaetes: some general constraints. Bull. Mar. Sci., v. 67, p. 433-447, 2000.

SCHLACHER, T. A.; DUGAN, J.; SCHOEMAN, D. S.; LASTRA, M.; JONES A.; SCAPINI, F.; McLACHLAN A.; DEFEO, O. Sandy beaches at the brink. Divers. Distrib., v. 13, p. 556-560, 2007.

SANTOS, P. J. P. Population dynamics and production of Scolelepis gaucha (Polychaeta: Spionidae) on the sandy beach of Southern Brazil. Mar. Ecol. Prog. Ser., v. 110, p. $159-165,1994$ 
SHIMIZU, R. M; RODRIGUES, S. A. Population ecology of Callichirus major (Say, 1818) (Crustacea: Decapoda: Thalassinidea) on a sandy beach in southeastern Brazil. In: KLEIN, J. C. V. V.; SCHRAM F. R. (Ed.). The biodiversity crisis and crustacean. In INTERNATIONAL CRUSTACEAN CONGRESS, 4 Amsterdam, 1998. Proceedings. p. 311-322 (Crustacean Issues 12).

SHORT, A. D. The role of wave weight, period, slope, tide range and embaymentisation in beach classifications: a review. Rev. Chil. Hist. Nat., v. 69, p. 589-604, 1996.

SOUZA, J. R. B.; BORZONE C. A. Population dynamics and secondary production of Scolelepis squamata (Polychaeta:Spionidae) in an exposed sandy beach of southern Brazil. Bull. Mar. Sci.,v. 67, p. 221-233, 2000.

SOUZA, J. R. B.; BORZONE C. A. A extração de corrupto, Callichirus major (Say) (Crustacea, Thalassinidae), para uso como isca em praias do litoral do Paraná: as populações exploradas. Revta. bras. Zool., v. 20, p. 625-630, 2003.

SOUZA, J. R. B.; BORZONE, C. A. Population dynamics and secondary production of Euzonus furciferus Ehlers (Polychaeta, Opheliidae) in an exposed sandy beach of Southern Brazil. Revta. bras. Zool., v. 24, p. 1139-1144, 2007.

SOUZA, J. R. B.; BORZONE, C. A.; BREY, T. Population dynamics and secondary production of Callichirus major (Crustacea: Thalassinidea) on a southern Brazilian sandy beach. Arch. Fish. Mar. Res., v.46, p. 151-164, 1998.

STITES, D. L.; BENKE A. Rapid growth rates of chironomids in three habitats of a subtropical blackwater river and their implications for P:B ratios. Limnol. Oceanogr., v. 34, p. 1278-1289, 1989.
TUMBIOLO, M. L.; DOWNING, J. A. An empirical model for the prediction of secondary production in marine benthic invertebrate populations. Mar. Ecol. Prog. Ser., v. 114, p.165-174, 1994.

VELOSO, V. G.; CARDOSO, R. S. Population biology of the mole crab Emerita brasiliensis (Decapoda:Hippidae) at Urca beach, Brazil. J. Crustacean Biol., v. 19, p.147$153,1999$.

VELOSO, V. G.; SALLORENZO, I. A. Differences in the secondary production of Emerita brasiliensis (Decapoda: Hippidae) on two sandy beaches in Rio de Janeiro State, Brazil. Nauplius, v.18, p. 57-68, 2010.

VELOSO V. G.; CARDOSO R. S.; PETRACCO M. Secondary production of the intertidal macrofauna of Prainha beach, Brazil. J. coast.Res., v. 15(SI), p. 385391, 2003.

WATERS, T. F. Secondary production in inland waters. Adv. Ecol. Res., v. 10, p. 91-164, 1977.

WILSON, J. G. Population dynamics and energy budget for a population of Donax variabilis (Say) on an exposed South Carolina beach. J. Exp. Mar. Biol. Ecol., v. 239, p. $61-83,1999$.

(Manuscript received 28 September 2011; revised 02 December 2012; accepted 03 December 2012) 